\title{
Steering-Enhanced Roughening during Metal Deposition at Grazing Incidence
}

\author{
Sebastiaan van Dijken, Louis C. Jorritsma,* and Bene Poelsema \\ Faculty of Applied Physics and Centre of Materials Research, University of Twente, P.O. Box 217, NL-7500 AE Enschede, The \\ Netherlands
}

(Received 11 December 1998)

\begin{abstract}
It is shown that steering may have an important influence on the morphology of growing films. Steering originates from long-range attractive forces between incoming atoms and substrate atoms and leads to preferential arrival of atoms on top of islands. This phenomenon is most pronounced for grazing incidence deposition and results in significantly increased roughness of the growing film. Steering, which is expected to be generally valid but has so far been disregarded in growth studies, is illustrated for the growth of $\mathrm{Cu} / \mathrm{Cu}(001)$. [S0031-9007(99)09145-0]
\end{abstract}

PACS numbers: 68.55.Jk, 61.14.Hg, 68.35.Bs, 81.10.-h

The evolution of nonequilibrium surfaces is a complex phenomenon, even for homoepitaxial growth systems. The emerging growth front is the result of roughening, due to the incident flux, which is counteracted by multiple transport processes tending to smoothen the surface. An important parameter in the balance between roughening and smoothing is the Ehrlich-Schwoebel (ES) barrier, which hinders downward diffusion across step edges $[1,2]$. A finite ES barrier results in a roughening of the growing surfaces [3-6], i.e., to the development of mounds.

Up to now, growth models have always regarded the flux of impinging atoms as being homogeneously distributed over the surface. Possible consequences of incident flux inhomogeneity as a result of surface roughness have been neglected completely. This shortcoming is remarkable since molecular dynamics calculations by Sanders et al. [7] show substantial deflection of nonnormal deposited atoms towards the surface, starting at about $1 \mathrm{~nm}$ above the outermost layer. This phenomenon can simply be rationalized since the attractive well, as seen by the approaching metal atoms, is of the order of a few eV's, while their kinetic energy typically amounts to only a few tenths of an eV. Consequently, the atoms must undergo strong acceleration towards the surface. In this Letter we report an effect which we believe is the first evidence for the importance of steering for the evolving morphology of the growth front. It gives rise to a remarkable heterogeneity of the incident flux, i.e., redistribution of the incident atoms due to their morphology dependent trajectories. Because of steering, atoms arrive preferentially on protruding terraces resulting in an enhanced roughening of the growing surface.

The experiments reported here have been conducted in ultrahigh vacuum (base pressure $<10^{-10}$ mbar) using spot profile analysis low energy electron diffraction (SPALEED). During growth, the temperature of the desulpherized $\mathrm{Cu}(001)$ substrate has been kept at $250 \mathrm{~K}$, while during measurements the temperature has been maintained at $100 \mathrm{~K}$. The deposited copper has been sublimated from an also desulpherized $\mathrm{Cu}$ disk, heated from the rear by means of electron bombardment. For the data discussed here the copper atoms have been deposited at a grazing angle of incidence $\left(80^{\circ}\right.$ from the normal) along the [110] azimuth. The homoepitaxial films have been grown at a rate of about 0.25 monolayers (ML) per minute. This deposition rate is about equal to that used in normal incidence experiments reported earlier [8], to which we will frequently refer here.

The central piece of data in this Letter is shown in Fig. 1: a profile of the specular beam obtained after deposition of 40 monolayers of copper on $\mathrm{Cu}(001)$ at grazing incidence. The fourfold symmetry, expected for the $\mathrm{Cu}(001)$ surface, and indeed measured after normal incidence deposition at similar conditions [8-11], is obliterated. Besides the dominant central Bragg peak two well developed facet peaks have emerged. Both peaks are positioned in the plane of incidence of the copper beam, while no clear diffraction features can be detected out of this plane. The observed diffraction pattern can be interpreted straightforwardly as resulting from growth induced parallel ripples at the $\mathrm{Cu}(001)$ surface, which are oriented perpendicular to the plane of incidence of the copper beam. The absence of out of plane diffraction features and the small out of plane width of the diffraction peaks suggest that these ripples are quite well defined and have an average length of about $50 \mathrm{~nm}$. The two clear in-plane facet peaks correspond to well established $\{1 \overline{1} 1\}$ and $\{1 \overline{1} 3\}$ facets on the illuminated and shadow sides of the ripples, respectively.

Compared to the facets which emerge after normal incidence deposition of 40 monolayers, the facets evolving at grazing incidence deposition are much better defined; i.e., their slope distribution is much narrower. In addition, the slopes of the illuminated and the shadow sides, corresponding to $\{1 \overline{1} 1\}$ and $\{1 \overline{1} 3\}$, respectively, are both substantially steeper than the average facet orientation of $(115)[8,12,13]$ obtained after normal incidence deposition at $250 \mathrm{~K}$. This illustrates the enhanced surface roughness after grazing incidence deposition. We attribute this enhanced roughness 
to the action of a basic phenomenon that until now has been discarded: redistribution of incident flux in favor of protruding surface areas due to a steering effect, which becomes substantial already at relatively large heights above the surface.

Steering is induced by long-range attractive forces between the substrate and the approaching slow copper atoms. Thermal copper atoms, approaching the surface at typical energies of $0.15 \mathrm{eV}$, experience a long-ranged attractive well of several eV's. This gives rise to substantial acceleration towards the surface. For the flat substrate this phenomenon has initially no consequences: the incident flux remains homogeneously distributed. The copper atoms only arrive at an effectively smaller polar angle of incidence. However, as soon as aggregates start to build up, the redistribution of material becomes progressively more important. To substantiate this phenomenon for a macroscopic surface we have performed a number of trajectory calculations. Being primarily interested in a qualitative (or semiquantitative) description, we have adopted a Lennard-Jones $(12,6)$ potential. Its parameters have been chosen such that both the copper interatomic distance as well as its cohesive energy are described quantitatively [14]. Trajectory calculations show that $150 \mathrm{meV}$ $\mathrm{Cu}$ atoms, directed with a grazing angle of incidence of $80^{\circ}$ towards the $\mathrm{Cu}(001)$ surface plane, do actually hit it at an angle of $17^{\circ}$. Clear deviations from the unaffected trajectories already occur at distances up to $1 \mathrm{~nm}$ above the outermost layer.

Figure 2(a) shows a cross-sectional view through the $\mathrm{Cu}(001)$ along the [110] azimuth. On this substrate we have constructed a monolayer high step in the direction perpendicular to the plane of incidence. This figure exhibits calculated equipotential energy contours (increment $-0.1 \mathrm{eV}$ ) as well as two calculated atom trajectories for atoms deposited with a grazing angle of $80^{\circ}$. The equipo-

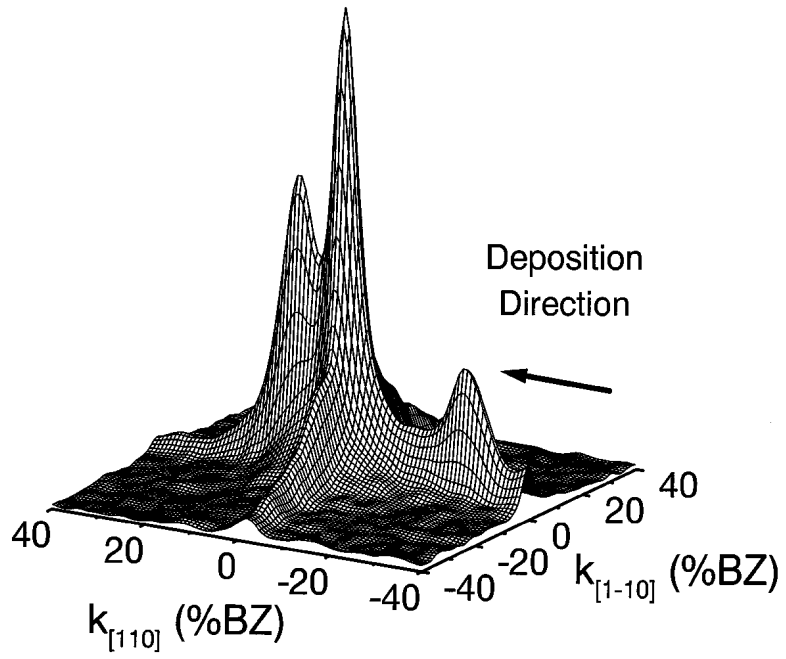

FIG. 1. SPA-LEED spot profile of the specular spot acquired after deposition of $40 \mathrm{ML} \mathrm{Cu}$ at an angle of $80^{\circ}$ from the surface normal with the substrate at $250 \mathrm{~K}$. tential energy contours show a pronounced step in the attractive potential, which extends laterally, e.g., for the $-0.01 \mathrm{eV}$ contour by as much as about $15 \AA$. Trajectory $B$ illustrates that, due to acceleration towards the surface, the incident atoms actually hit the surface quite a long distance $\left(\delta_{B}\right)$ before their target point. The target point is the intersection of the asymptotic long distance part of the trajectory with the surface. Far away from the step in the attractive potential $\delta$ is constant and irrespective of the level at which the atoms arrive. Consequently, a homogeneous flux is expected. Consider now the situation as illustrated by trajectory $A$ : When the atom passes the island

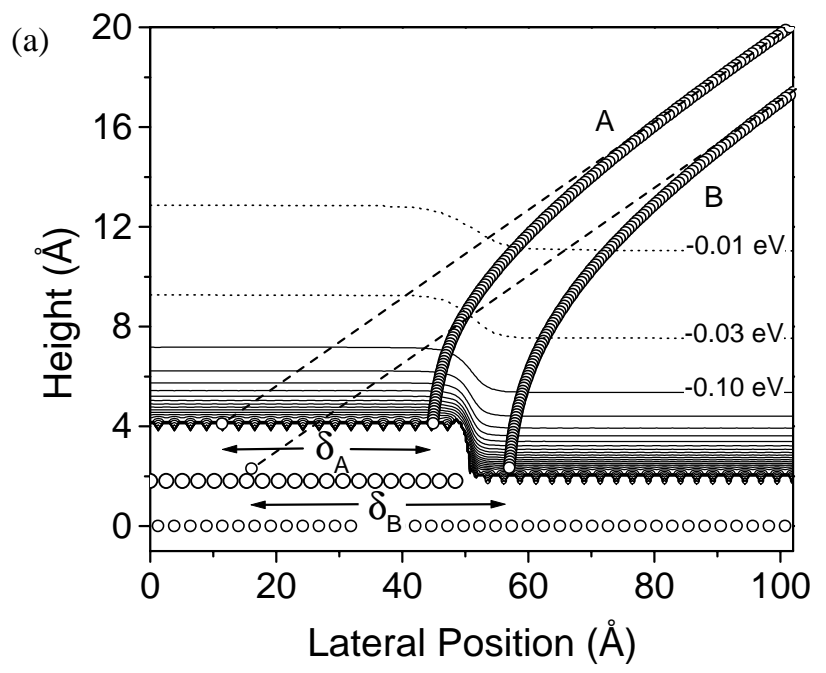

(b)

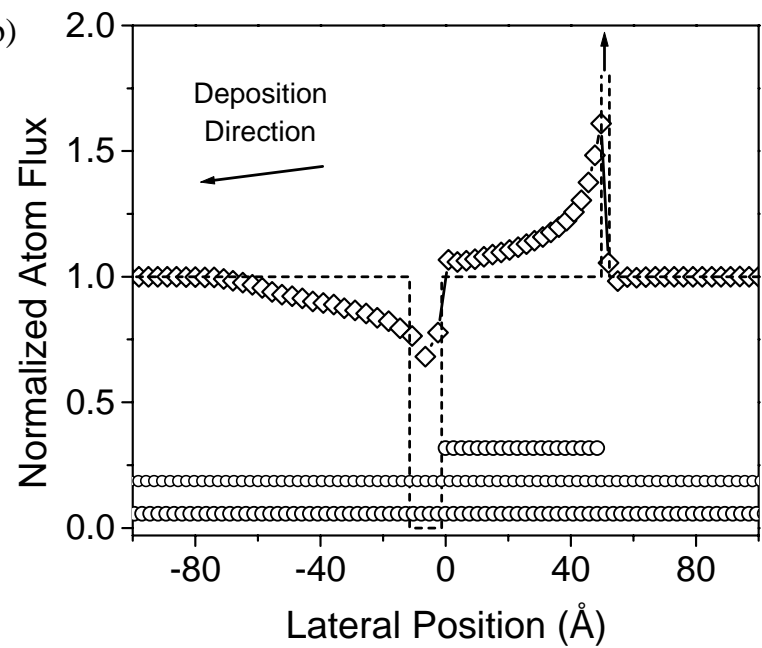

FIG. 2. (a) Calculated equipotential energy contours and two atom trajectories for a surface with a step edge in the direction perpendicular to the plane of incidence (note the different length scale on the two axes). The increase in attractive potential is $-0.1 \mathrm{eV}$ for the solid contour lines. The trajectory calculations for a deposition angle of $80^{\circ}$ (with respect to the surface normal) start at $20 \AA$ above the surface. (b) Calculated adatom flux at the surface, normalized to a homogeneous atom flux far above the surface. The dashed line shows schematically the adatom flux in the case of classical shadowing without steering. 
boundary it suddenly experiences the surface at a much smaller distance and impact is closer. Consequently, the value of $\delta_{A}$ is reduced giving rise to a local enhancement of the incident atom flux just behind the front edge of the island as illustrated in Fig. 2(b). In fact all arriving atoms whose trajectories pass through areas of substantial distortion of the attractive potential related to the ascending step contribute to enhanced flux. They are focused onto the near step region. We refer to this phenomenon as steering. For the 1 monolayer high island [see Fig. 2(b)] the enhancement factor amounts to about 1.6 at the front of the island and decreases to unity going further downstream on an extended island. Behind the island the density of the incident atoms is reduced, which is related to the shadowing by the island. The reduction of flux behind the island compensates the flux enhancement on top of the island as should be the case for particle conservation reasons. Note that the flux behind the island never becomes zero, which would be the case for classical shadowing without steering. For comparison, the classical situation is also drawn in Fig. 2(b). In this case no flux enhancement on top of the island is observed: the flux missing in the shadow zone is concentrated in front of the illuminated side of the island. So we clearly obtain a redistribution of incident flux, related to steering of the atoms towards the top of the growing island. The effect being substantial already for monolayer high adatom islands strongly gains importance when the surface becomes rougher. Calculations of the normalized incident atom flux for a 3 monolayer high adstructure show enhancement factors well exceeding three. This phenomenon obviously leads to rougher growth fronts [15], which is enhanced by the presence of a finite ES barrier. We note in addition that the range of the shadow region is much smaller for the classical shadowing case than in the presence of steering. This phenomenon contributes to improved lateral order. Indeed we observe a narrow separation distribution of the ripples in the plane of incidence [16].

Already in the submonolayer regime the growth behavior is affected by the deposition geometry. This statement is illustrated nicely in Fig. 3 showing the specular beam profile obtained after grazing incidence deposition of about 0.5 ML. The narrow Bragg peak is surrounded by a ring, the position of which is given by the distance between the adatom islands. This quasidiffuse scattering ring has perfect rotational symmetry after normal incidence deposition [8]. In the present case of grazing incidence deposition, however, the ring intensity exhibits a clearly developed twofold symmetry. It possesses two pronounced minima, in the direction perpendicular to the plane of incidence. In fact, the development of a twofold symmetric ring is already visible after deposition of 0.3 monolayers copper. This remarkable beam profile reveals the presence of homogeneously distributed rectangular islands in contrast to the square ones growing at normal in- cidence. The step edges remain oriented along the close packed $\langle 110\rangle$ azimuths. Approaching the energetic preference for $\langle 110\rangle$ ledges is facilitated by sufficient ledge adatom diffusion at the considered substrate temperatures [17]. A comparison with calculations (kinematic approximation) indicates that the rectangular islands have an aspect ratio of about 1.05. They would have been hardly noticed with STM. The applied diffraction method is well able to distinguish this feature. The long sides of the islands are oriented perpendicular to the plane of incidence of the deposited $\mathrm{Cu}$ atoms.

The development of rectangular islands at submonolayer coverages turns out to provide a welcome and independent check of the consistency of the model conclusions derived above. From the fact that homogeneously distributed square islands develop during normal deposition we learn that the diffusion of copper on $\mathrm{Cu}(001)$ is isotropic and ledge adatom diffusion is readily active at $250 \mathrm{~K}$. This implies that the evolution of rectangular islands has to be related to peculiarities connected with the grazing incidence of the copper atoms. In particular, the total step edge advance rate perpendicular to the plane of incidence has to be slightly higher than the sum of the advance rates of the illuminated edge and the shadow edge of the adatom islands. Let us consider first the situation without steering. If the arriving atoms are instantaneously equilibrated the step edge advance rate on the shadow side is strongly reduced since its capture zone receives less atoms than in the case of normal incidence. On the basis of particle conservation this feature is exactly compensated by the larger impingement rate in the capture zone of the illuminated step edge. Consequently, the sums of the in-plane and of the out-of-plane step edge advance rates are exactly equal. Since in the considered case (there is no attractive potential and atoms stick where they hit the surface) there is no reason for an anisotropic descent of atoms from the top of the adatom island, square islands

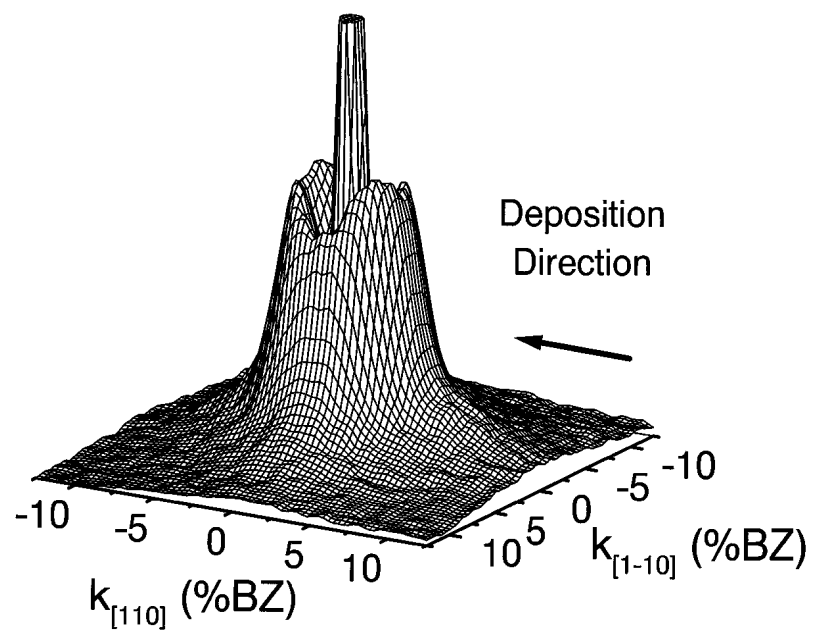

FIG. 3. Profile of the specular SPA-LEED peak acquired after deposition of $0.5 \mathrm{ML} \mathrm{Cu}$ at $80^{\circ}$ with the substrate at $250 \mathrm{~K}$. 
must develop. If, however, we switch on steering, the anisotropic shapes can be explained. Steering will lead to a redistribution of incident flux to the top of the adatom islands: flux that without steering would contribute to an upstream advance of the illuminated step edge now lands on top of the island and is partly descending across the [110] oriented ledges. This gives rise to the evolution of rectangular islands (aspect ratio $\approx 1.05$ ), which are elongated perpendicular to the plane of incidence. Estimates of the magnitude of this feature lead to values well consistent with the experimental data.

We finally note that steering should be anticipated in any growth situation. Its consequences may vary from insignificant for monolayer high islands at normal deposition to substantial at grazing incidence. Steering is expected to be stronger for metals than for semiconductors or isolators. In general, it will be strong when substrate and incident atoms have relatively high electronic polarizabilities. The well established small but nonzero ES barrier for $\mathrm{Cu} / \mathrm{Cu}(001)[8,10,11]$ leads to significant effects. The consequences should be even more substantial for higher values of the ES barrier, which are commonly related to close-packed [e.g., fcc(111)] metal surfaces.

Summarizing, the presented grazing incidence growth experiments of $\mathrm{Cu} / \mathrm{Cu}(001)$ provide unambiguous evidence for the importance of steering for the morphology of the growth front. Steering leads to a substantial redistribution of incident flux and in fact to focusing of the incident particles onto the uppermost levels of adatom aggregates. This so far unanticipated phenomenon in epitaxial growth leads to enhanced roughness of the growth front, in particular, when the incident particles are deposited at grazing incidence. When comparing experiments mutually or to calculations the exact deposition geometry must be considered. In particular, during the emergence of growth induced facets the above discussed steering effect may become of prime importance (Ref. [8], and references therein). Grazing incidence deposition of copper on $\mathrm{Cu}(001)$ leads to the emergence of highly ordered arrays of parallel asymmetric ripples oriented normal to the plane of incidence. This novel observation may well be used to structure substrates, which may subsequently act as templates for heteroepitaxial structures.
We gratefully acknowledge useful comments and critical reading of the manuscript by Georg Rosenfeld, Herbert Wormeester, and Harold Zandvliet.

*Present address: ASML, P.O. Box 324, NL-5500 AH Veldhoven, The Netherlands.

[1] G. Ehrlich and F. G. Hudda, J. Chem. Phys. 44, 1039 (1966).

[2] R. L. Schwoebel and E. J. Shipsey, J. Appl. Phys. 37, 3682 (1966).

[3] R. Kunkel, B. Poelsema, L. K. Verheij, and G. Comsa, Phys. Rev. Lett. 65, 733 (1990).

[4] J. Villain, J. Phys. I 1, 19 (1991).

[5] K. Meinel, M. Klaua, and H. Bethge, J. Cryst. Growth 89, 447 (1988).

[6] B. Poelsema, R. Kunkel, L. K. Verheij, and G. Comsa, Phys. Rev. B 41, 11609 (1990).

[7] D. E. Sanders, D. M. Halstead, and A. E. DePristo, J. Vac. Sci. Technol. A 10, 1986 (1992).

[8] L.C. Jorritsma, M. Bijnagte, G. Rosenfeld, and B. Poelsema, Phys. Rev. Lett. 78, 911 (1997).

[9] G. L. Nyberg, M. T. Kief, and W.F. Egelhoff, Jr., Phys. Rev. B 48, 14509 (1993).

[10] H. Dürr, J.F. Wendelken, and J.-K. Zuo, Surf. Sci. 328, L527 (1995).

[11] J.-K. Zuo and J. F. Wendelken, Phys. Rev. Lett. 78, 2791 (1997).

[12] H.-J. Ernst, F. Fabre, R. Folkerts, and J. Lapujoulade, Phys. Rev. Lett. 72, 112 (1994).

[13] H.-J. Ernst, F. Fabre, R. Folkerts, and J. Lapujoulade, J. Vac. Sci. Technol. A 12, 1809 (1994).

[14] The used potential is almost identical to the one denoted with LJ-C in the paper by D.E. Sanders and A.E. DePristo [Surf. Sci. 254, 341 (1991)]. The authors found the atom trajectories for $\mathrm{Cu}$ approaching $\mathrm{Cu}(001)$ satisfactorily close to those calculated with their most accurate many body density functional based potential energy surface.

[15] G. Rosenfeld, B. Poelsema, and G. Comsa, J. Cryst. Growth 151, 230 (1995).

[16] S. van Dijken and B. Poelsema (to be published).

[17] M. Breeman, G. T. Barkema, and D. O. Boerma, Surf. Sci. 303, 25 (1994). 